\title{
Liquid core fibre Bragg grating based refractive index sensor formed by femtosecond assisted chemical etching technique
}

\author{
P. Saffari,"Z. Yan, K. Zhou, L. Zhang, and I. Bennion \\ Photonics Research Group, School of Engineering and Applies Science, Aston University, \\ Birmingham, B4 7ET UK \\ *Corresponding author: saffarip@aston.ac.uk
}

\begin{abstract}
We report the fabrication of a refractive index (RI) sensor based on a liquid core fibre Bragg grating (FBG). A micro-slot FBG was created in standard telecom optical fibre employing the tightly focused femtosecond laser inscription aided chemical etching. A micro-slot with dimensions of 5.74(h) $\times 125(\mathrm{w}) \times 1388.72(\mathrm{l}) \mathrm{m}$ was engraved across the whole fibre and along $1 \mathrm{~mm}$ long FBG which gives advantage of a relatively robust liquid core waveguide. The device performed the refractive index sensitivity up to about $742.72 \mathrm{~nm} / \mathrm{RIU}$.
\end{abstract}

Keywords: Fibre Bragg grating, Refractive index sensor, Femtosecond laser inscription, Chemical etching

\section{INTRODUCTION}

FBGs of normal structure cannot be used as RI sensor directly as the mode coupling takes place only within the core modes which are screened by the cladding from surrounding medium. However, modified FBGs have been proposed and demonstrated as RI sensors, including (a) forward cladding mode coupling in tilted fibre gratings (TFGs) with large tilt angles ${ }^{1}$, (b) backward cladding mode coupling generated by tilted FBGs with small tilt angles ${ }^{2}$ and (c) backward core mode coupling in FBGs with modified structures in which the fiber cladding being fully removed ${ }^{3}$. Between the mentioned categories, TFGs with large angle structure are the most RI sensitive devices but they are limited in respect of miniaturisation because of their long size and also use of transmission detection. For small angle TFGs, the RI sensitivity is very low and the FBGs with removed cladding are extremely fragile to use. In all three categories, only a small portion of the propagating light exists in the cladding to interact with the surrounding medium, thus suffering from the limited sensitivity and an operational range below the index of fiber core. Another reported approach to enhance the RI sensitivity is to employ a liquid as the core of the waveguide instead of its cladding to guide the light in applications, such as spectroscopy ${ }^{5}$. For the first time, Zhou et.al reported a fibre based liquid core device employing a micro-slot grating to interrogate the surrounding medium refractive index (SRI) ${ }^{4}$. He employed the femtosecond (fs) laser inscription assisted chemical etching technique to engrave a micro-slot of $1.2 \times 125 \times 50$ qum along a UV inscribed FBG. Here we report the further development on this device by inscribing a microslot with dimensions of $5.74 \times 125 \times 1388.74 \mathrm{~lm} \mathrm{w}$ hich across the whole length of a $1 \mathrm{~mm}-\mathrm{long}$ FBG and the characteristics of the fabricated device in terms of SRI and temperature sensitivities.

\section{SAMPLE FABRICATION}

Figure.1 presents the geometry of the proposed micro-slot engraved in the core of the fibre and along with an FBG. Fabrication process of the proposed micro-slot in FBG includes three main steps: (a) fabricating FBG into the core of a hydrogen-loaded standard telecom single-mode fibre by UV light using the phase mask inscription technique;(b) patterning the micro-slot of the desired dimension along the full length of FBG and across the whole fibre using tightly focused fs laser beam and (c) etching the fs-modified FBG in a 5\% hydrofluoric acid (HF) solution for selective removal of the fs-modified

21st International Conference on Optical Fiber Sensors, edited by Wojtek J. Bock, Jacques Albert, Xiaoyi Bao, Proc. of SPIE Vol. 7753, 77538P · C 2011 SPIE · CCC code: 0277-786X/11/\$18 · doi: 10.1117/12.885086 
region. 1mm-long FBG with the central wavelength of $1552.25 \mathrm{~nm}$ and reflectivity of $\sim 9 \mathrm{~dB}$ was UV inscribed first and then annealed for 48 hours at $103 \mathrm{C}$ to become stabilised. The temperature sensitivity of $11.5 \mathrm{pm} /{ }^{\circ} \mathrm{C}$ for the fabricated FBG was measured at this stage. After that, a $125(\mathrm{w}) \times 5.74(\mathrm{~h}) \times 1388.72(\mathrm{l}) \mathrm{lm}$ micro - -slot was patterned along the FBG across the whole fibre by employing the fs-laser inscription technique. The fs-laser pulses of $\lambda=800 \mathrm{~nm}$ were tightly focused on the fibre by a $\times 100$ objective lens with N.A. of 0.55 while the working distance was set at $13 \mathrm{~mm}$. The pulse width of the laser was $\sim 150$ fs with $1 \mathrm{kHz}$ repetition rate. The fibre was mounted on a dual-axis air-bearing translation stage to write the desired pattern simply by translating the fibre with respect to the fs-laser beam. Following this step, the fibre with the microslot pattern was chemically etched in 5\% HF acid solution and an ultrasonic bath was employed to increase the penetration of HF acid into the patterned region of the fibre. The fs-laser patterning caused a very high etching rate which was approximately 100 times higher than in a pristine fiber. After approximately 15 minutes of chemical etching, a micro-slot with a height of $5.74 \mu \mathrm{m}$ was generated along the FBG and across the fibre. The microscope images of the proposed device under $\times 5$ and $\times 40$ microscopic lenses are presented in figure. 2 .

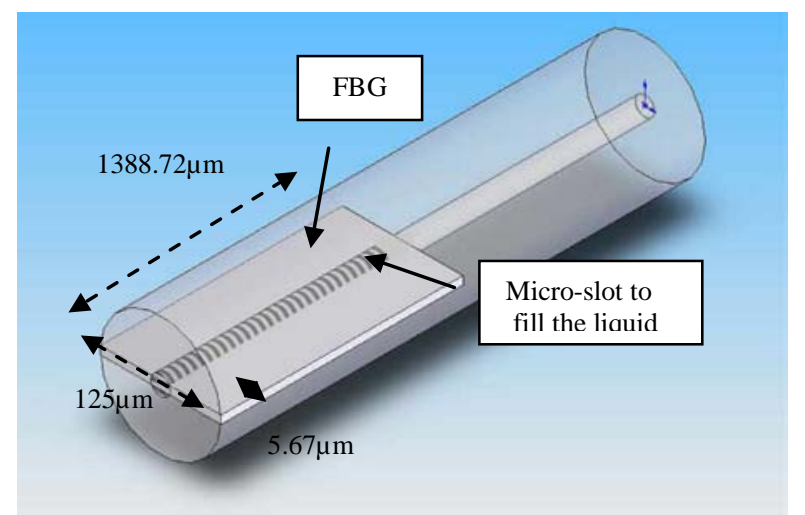

Figure 1. The geometry of the proposed micro-slot along an FBG and across the fibre

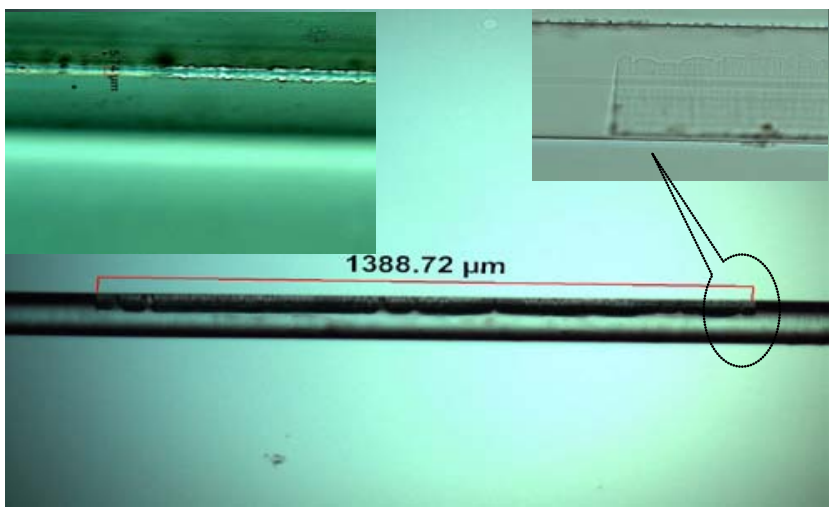

Figure 2. Microscope image under a $\times 5$ microscopic lens. The insets are the microscope images of the same device observed by $\times 40$ oil-immersion microscopic lens.

\section{SAMPLE CHARACTRESTICS}

Transmission loss profile of the micro-slot FBG device was analysed by using an optical vector analyser (EL from LUNA Technologies). When the sample was surrounded by air, Fabry-Perot resonance features spread all over the spectrum and the original Bragg peak was not observed as the core refractive index was reduced significantly because the induced air-filled micro-slot occupying a relatively large area of the fiber core where the grating was located. Then, we applied index gel of RI 1.36 on the sample and examined the transmission spectrum again (figure 3). With the micro-slot filled by the index gel, the Bragg peak appeared in the spectrum but at lower wavelength than the original FBG. Here the Bragg peak is located at $1548.39 \mathrm{~nm}$ with a $1 \mathrm{~dB}$ attenuation band while in the original FBG without the micro-slot, the Bragg peak was at 1552.25nm and exhibiting $9 \mathrm{~dB}$ attenuation. This data evidently present that the loss is induced by the big micro-slot across the fibre and because the total effective index of the core is lower, so the Bragg wavelength shifts to shorter wavelength side.

When the sample was filled with index gel of $1.478 \mathrm{RI}$ gel which is higher than the original core, we observed the appearance of one Bragg peak at 1551.21nm close to its original wavelength and two more Bragg reflection peaks located in higher wavelength range, as shown in figure 4. The multi Bragg reflection peaks indicate the effective index is high in the combined core region, thus the fibre becomes multi-mode. 


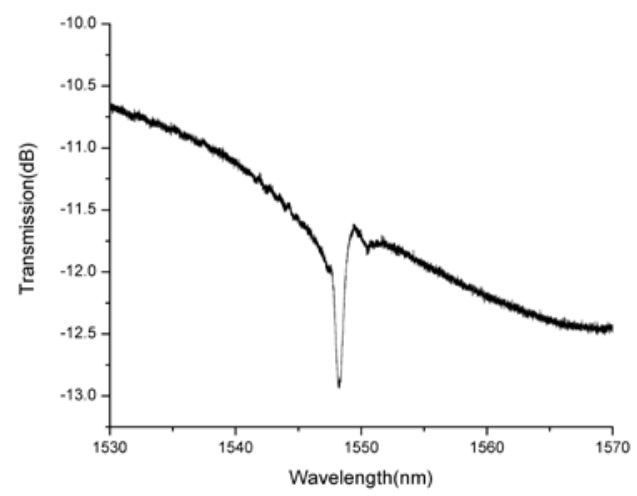

Figure 3. Transmission loss profile of the micro-slot FBG when it was immersed in index gel of $1.36 \mathrm{RI}$.

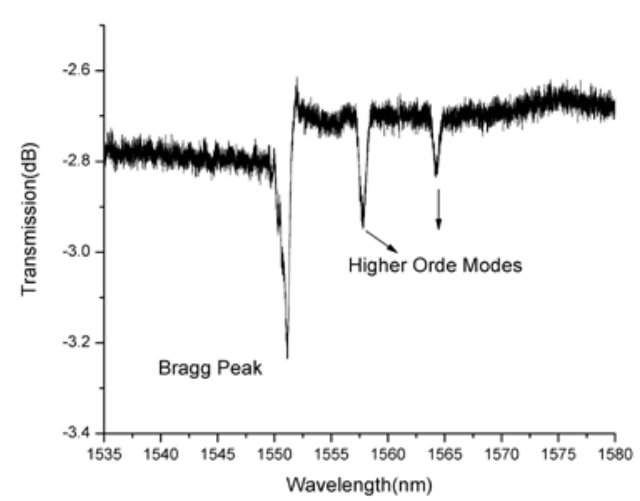

Figure 4. Transmission loss profile of the proposed device when it was immersed in index gel of 1.478 RI.

The RI response of the proposed micro-slot FBG device was investigated by applying a set of index gels (from Cargille Laboratories) on the sample while the transmission spectrum of the device for each individual RI gel was measured. In this experiment, the device was fixed in a V-grooved aluminum plate to ensure the measurement was taken free from other effects including strain, bend and temperature. Considering the relatively big size of the micro-slot and ensuring no residue RI gel left in the micro-lot after applying each RI gel, the sample was rinsed with acetone, methanol and water after each measurement. This process was repeated for several times until the original spectrum of the device when surrounded by air was restored and the sample was ready for the next RI measurement. Figure 5 indicates the RI response of the micro-slot FBG in a range from 1.36 to 1.478. This figure indicates two very different response regions for this sample. In the low RI region from 1.36 to 1.40 , the peak around $1548 \mathrm{~nm}$ is almost insensitive to the applied RI while for the high RI values from 1.40 to 1.496 , the new peak at longer wavelength $(\sim 1550 \mathrm{~nm})$ exhibits the RI sensitivity of about $742.72 \mathrm{~nm} / \mathrm{RIU}$. It can be explained that in the low RI region, the launched light is weakly confined, so that the effective index of the core is more dependent on the glass, while for sensing of high RI, the light is more guided in the liquid core, and eventually leading to forming multi-mode structure with their majority of the light confined in the liquid and thus their index changes are much more dependent on the sensing liquid. This result is in a good agreement with the reported simulation and experimental results in Zhou.et.al paper ${ }^{4}$.

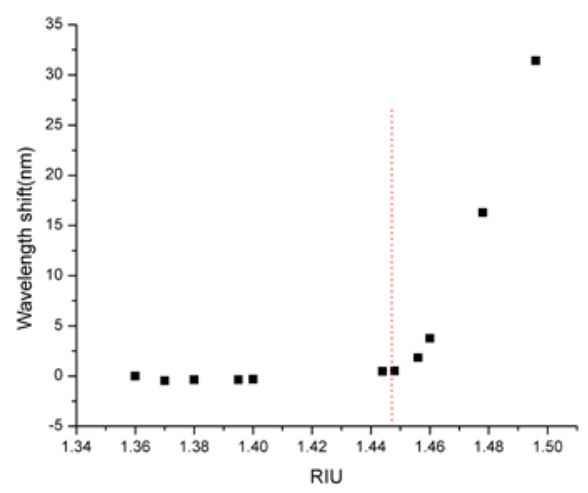

Figure 5. RI characteristics of the proposed micro-slot FBG device

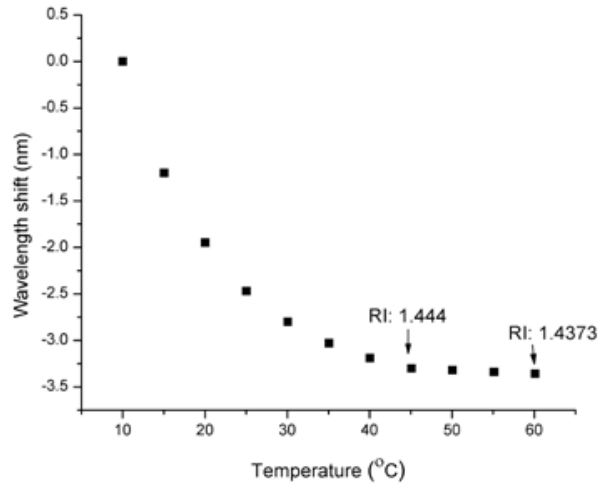

Figure 6. Thermal response of the micro-slot FBG device when it was immersed in $1.456 \mathrm{RI}$ gel. 
The temperature sensitivity of the proposed device was also measured when it was subjected to index gel of $1.456 \mathrm{RI}$ and the result is presented in figure 6. This figure clearly indicates an exponential thermal decay response from the device. Here, also there are two well separated response regions corresponding to the low (from $10^{\circ} \mathrm{C}$ to $45^{\circ} \mathrm{C}$ ) and high $\left(45^{\circ} \mathrm{C}\right.$ to $60^{\circ} \mathrm{C}$ ) temperature values.

Due to the negative thermo-optic coefficient effect of liquid, the refractive index of the gel is reduced by enhancing the temperature. The rate of this decrease for this typical 1.456 RI gel is determined by the value of

$$
-\frac{d n}{d t}=3.74 \times 10^{-4 c} \quad \text { (The value has been provided by Cargille Laboratories). }
$$

The calculation based on this factor presents that by increasing the temperature to $45^{\circ} \mathrm{C}$, the RI value of the gel reduces to 1.444 and when the temperature is set at $60^{\circ} \mathrm{C}$ this value further reduces to 1.4373 . In the low temperature response range, the RI of the gel changes from 1.456 to 1.444. Similar to the given explanation for the investigated RI response of the device, the gel is a leading factor and the Bragg peak shifts to the shorter wavelength due to the decreasing to RI of the gel. After the temperature further increases above $45^{\circ} \mathrm{C}$, the RI value of the index gel becomes close to the glass so the downward trend of the Bragg wavelength shift stops. At this point since the sample is about to start behaving with a core refractive index very close to fibre glass, it is predicted that by further increase in temperature, we should observe a linear red-shift of the Bragg peak of this micro-slot FBG device.

\section{CONCLUSION}

In conclusion, we have experimentally proved that the proposed micro-slot FBG is effective to sense and monitor refractive index of liquids in a broad RI range from 1.40 to 1.496 with the sensitivity as high as $742.72 \mathrm{~nm} / \mathrm{RIU}$. The thermal response of the device was measured to be about $7.3 \mathrm{pm} /{ }^{\circ} \mathrm{C}$ while it was surrounded by air. The temperature measurement was also carried out for this device while it was immersed in RI gels and the result evidently shows that when the temperature increases, the thermal response of the micro-slot FBG decreases due to the decreasing of the RI value of the gel. Overall, the proposed micro-slot FBG structure exhibited not only the enhanced sensitivity to the external RI, but also a better mechanical strength than other chemically etched FBG sensing devices, thus represent a more ideal structure for implementation of infiber bio-chemical sensors.

\section{REFERENCES}

[1]. Zhou. K, Zhang. L, Chen. X and Bennion. I, "Optic sensors of high refractive-index responsivity and low thermal cross sensitivity that use fiber Bragg gratings of $>80^{\circ}$ tilted structures", Opt. Lett, 31, 1193-1195(2006).

[2]. Chan. C. F, Chen. C, Jafari. A, Laronche. A, Thomson. D. J and Albert. J, "Optical fiber refractometer using narrowband cladding-mode resonance shifts", Appl.Opt, 46, 1142-1149 (2007).

[3]. Iadicicco. A, Cusano. A, Cutolo. A, Bernini. R and Giordano. M, "Thinned fiber Bragg gratings as high sensitivity refractive index sensors”, IEEE Photon. Technol. Lett, 16, no. 4, pp. 1149-1151(2004).

[3]. Zhou. K, Lai. Y, Chen. X, Sugden. K, Zhang.L and Bennion. I, "A refractometer based on a micro-slot in a fiber Bragg grating formed by chemically assisted femtosecond laser processing”, Opt. Express, 15, no. 24, pp. 15848-15853(2007).

[4]. Holtz. M, Dasgupta. P. K and Zhang. G, "Small-volume Raman spectroscopy with a liquid core waveguide", Anal. Chem, 71, 2934 -2938 (1999). 